\title{
Interference between the atmospheric and solar oscillation amplitudes
}

\author{
Patrick Huber, ${ }^{\ddagger}$ Hisakazu Minakata $\odot,{ }^{*}$ and Rebekah Pestes $\odot^{\dagger}$ \\ Center for Neutrino Physics, Department of Physics, Virginia Tech, Blacksburg, Virginia 24061, USA
}

(Received 17 December 2019; accepted 22 April 2020; published 12 May 2020)

\begin{abstract}
We propose to detect the interference effect between the atmospheric-scale and solar-scale waves of neutrino oscillation, one of the key consequences of the three-generation structure of leptons. In vacuum, we show that there is a natural and general way of decomposing the oscillation amplitude into these two oscillation modes. The nature of the interference is cleanest in the $\bar{\nu}_{e}$ disappearance channel, since it is free from the $C P$ phase $\delta$. We find that the upcoming JUNO experiment offers an ideal setting to observe this interference with more than $4 \sigma$ significance, even under conservative assumptions about the systematic uncertainties. Finally, we discuss the relationship between the mass-ordering resolution and the interference effect.
\end{abstract}

DOI: 10.1103/PhysRevD.101.093002

\section{INTRODUCTION}

It is a remarkable feature of nature that the fundamental fermions, quarks, and leptons come into our world in the form of three generations, which has various important consequences. The most dramatic one among them would be to provide a mechanism for $C P$ violation [1]. The Standard Model of particle physics with three families of quarks, not two, allows for the existence of a phase, the Kobayashi-Maskawa (KM) phase [2], by which $C P$ symmetry is broken; and indeed, $C P$ violation has been observed [3-5]. If a similar phase exists in the lepton sector in the neutrino-mass-embedded Standard Model $(\nu \mathrm{SM})$, then there will be $C P$ violation due to the lepton KM phase. ${ }^{1}$ The observation of the leptonic $C P$ phase is being and will be actively pursued by the ongoing and nextgeneration neutrino oscillation experiments [7-11].

It is a natural question whether $C P$ violation is the only consequence of the three-family structure. In the context of neutrino oscillation, the three-generation structure allows for the existence of two independent mass-squared differences: $\Delta m_{31}^{2} \equiv m_{3}^{2}-m_{1}^{2}$ and $\Delta m_{21}^{2}$. Experimentally, we find the $\Delta m_{31}^{2}$-driven atmospheric neutrino oscillation [12] and the solar $\Delta m_{21}^{2}$-driven reactor neutrino oscillation

*minakata71@vt.edu

†rebhawk8@vt.edu

"pahuber@vt.edu

${ }^{1}$ Note that if neutrinos are Majorana particles [6], there is an option of having $C P$ violation with only two generations of leptons.

Published by the American Physical Society under the terms of the Creative Commons Attribution 4.0 International license. Further distribution of this work must maintain attribution to the author(s) and the published article's title, journal citation, and DOI. Funded by SCOAP ${ }^{3}$.
[13], as well as the $\Delta m_{21}^{2}$-matter potential induced flavor conversion $[14,15]$ inside the Sun [16].

Building on this success, in this paper, we wish to add a new item to the list of nontrivial consequences of the threegeneration structure: Quantum interference between the atmospheric-scale and solar-scale waves of neutrino oscillation. So far, the existence of the small $\Delta m_{21}^{2}$ effects in atmospheric and long-baseline (LBL) accelerator neutrino experiments and, similarly, the effects of the larger $\Delta m_{31}^{2}$, as well as the $\theta_{13}$ mixing effect, in the solar neutrino observation have been recognized as small subleading effects. The simultaneous full existence of both the $\Delta m_{31}^{2}$ and $\Delta m_{21}^{2}$ waves and their mutual interference, if observed, would establish another consequence of the three-generation structure of neutrinos embedded into the $\nu \mathrm{SM}$. For previous discussions which addressed related interference phenomena, see, e.g., Refs. [17,18] for accelerator/atmospheric neutrinos, and Refs. [19-21] for reactor neutrinos.

\section{THE ATMOSPHERIC AND SOLAR AMPLITUDES}

Our first task is to define what the atmospheric and solar amplitudes are in neutrino oscillation. In this paper, we restrict our discussion to vacuum, as a similar generic definition fulfilling the conditions 1 and 2 below is not available - in fact, very likely not existing — in matter [22]. ${ }^{2}$ The flavor-basis $S$-matrix elements $S_{\alpha \beta}(\alpha, \beta=e, \mu, \tau)$, which describe the neutrino flavor transformation $\nu_{\beta} \rightarrow \nu_{\alpha}$,

\footnotetext{
${ }^{2}$ The authors of Ref. [18] propose a particular way of decomposition into the "atmospheric" and "solar" amplitudes in matter. We will contrast their method to our own proposal in Ref. [22].
} 
can be written under the ultrarelativistic approximation of neutrinos as

$S_{\alpha \beta}=U_{\alpha 1} U_{\beta 1}^{*}+U_{\alpha 2} U_{\beta 2}^{*} e^{-i \frac{\Delta m_{21}^{2}}{2 E} x}+U_{\alpha 3} U_{\beta 3}^{*} e^{-i \frac{\Delta m_{31}^{2}}{2 E} x}$,

where $E$ is the energy and $\Delta m_{j i}^{2} \equiv m_{j}^{2}-m_{i}^{2}(i, j=1,2,3)$ denotes the mass-squared differences of neutrinos. $U_{\alpha i}$ is the element of the lepton flavor mixing matrix which relates the flavor and the mass eigenstates of neutrinos as $\nu_{\alpha}=U_{\alpha i} \nu_{i}$. In Eq. (1), we factor out $e^{-i m_{1}^{2} x / 2 E}$ for simplicity of the expression, which of course does not alter the physical observables. The oscillation probability of the process $\nu_{\beta} \rightarrow \nu_{\alpha}$ is given by $P\left(\nu_{\beta} \rightarrow \nu_{\alpha}: x\right)=\left|S_{\alpha \beta}\right|^{2}$. Hereafter, again for simplicity of the expressions, we define

$$
\Delta_{j i} \equiv \frac{\Delta m_{j i}^{2}}{2 E}
$$

We take a heuristic way to find the appropriate definitions of the atmospheric and solar amplitudes. Let us first discuss the appearance channel, $\alpha \neq \beta$. The $S$-matrix elements in Eq. (1) can be rewritten as

$$
S_{\alpha \beta}=U_{\alpha 3} U_{\beta 3}^{*}\left(e^{-i \Delta_{31} x}-1\right)+U_{\alpha 2} U_{\beta 2}^{*}\left(e^{-i \Delta_{21} x}-1\right)
$$

due to unitarity, $U_{\alpha 1} U_{\beta 1}^{*}+U_{\alpha 2} U_{\beta 2}^{*}+U_{\alpha 3} U_{\beta 3}^{*}=0$. Then, we claim that

$$
S_{\alpha \beta}^{\mathrm{atm}} \equiv U_{\alpha 3} U_{\beta 3}^{*}\left(e^{-i \Delta_{31} x}-1\right)
$$

is the atmospheric amplitude, and

$$
S_{\alpha \beta}^{\mathrm{sol}} \equiv U_{\alpha 2} U_{\beta 2}^{*}\left(e^{-i \Delta_{21} x}-1\right)
$$

is the solar amplitude. The atmospheric amplitude, by definition, describes neutrino oscillation due to nonvanishing $\Delta m_{31}^{2}$, and the solar amplitude describes the oscillation caused by $\Delta m_{21}^{2}$. Therefore, the obtained expressions (4) and (5) for them are entirely natural ones.

In disappearance channels, due to a difference in unitarity, $U_{\alpha 1} U_{\alpha 1}^{*}+U_{\alpha 2} U_{\alpha 2}^{*}+U_{\alpha 3} U_{\alpha 3}^{*}=1$, the $S$ matrix has a slightly different expression when it is written in terms of the atmospheric and the solar amplitudes,

$$
\begin{aligned}
S_{\alpha \alpha} & =1+\left|U_{\alpha 3}\right|^{2}\left(e^{-i \Delta_{31} x}-1\right)+\left|U_{\alpha 2}\right|^{2}\left(e^{-i \Delta_{21} x}-1\right) \\
& =1+S_{\alpha \alpha}^{\mathrm{atm}}+S_{\alpha \alpha}^{\mathrm{sol}},
\end{aligned}
$$

where $S_{\alpha \alpha}^{\text {atm }}$ and $S_{\alpha \alpha}^{\text {sol }}$ are defined by extending the definition in Eqs. (4) and (5), by setting $\beta=\alpha$. They, of course, satisfy the conditions $S_{\alpha \alpha}^{\text {atm }} \rightarrow 0$ when $\Delta_{31} \rightarrow 0$, and $S_{\alpha \alpha}^{\text {sol }} \rightarrow 0$ when $\Delta_{21} \rightarrow 0$, respectively.
Now, we try to elevate the heuristic definitions into the general definition of $S_{\alpha \beta}^{\text {atm }}$ and $S_{\alpha \beta}^{\text {sol }}$. For a given $S$-matrix element $S_{\alpha \beta}$,

(1) The atmospheric and the solar amplitudes are defined, respectively, as

$$
S_{\alpha \beta}^{\mathrm{atm}}=\lim _{\Delta m_{21}^{2} \rightarrow 0} S_{\alpha \beta}, \quad S_{\alpha \beta}^{\mathrm{sol}}=\lim _{\Delta m_{31}^{2} \rightarrow 0} S_{\alpha \beta} .
$$

(2) We demand the completeness condition $S_{\alpha \beta}=$ $\delta_{\alpha \beta}+S_{\alpha \beta}^{\mathrm{atm}}+S_{\alpha \beta}^{\mathrm{sol}}$,

where $\delta_{\alpha \beta}$ denotes the Kronecker delta function. Consistency requires the so-obtained amplitudes to satisfy $\lim _{\Delta m_{31}^{2} \rightarrow 0} S_{\alpha \beta}^{\text {atm }}=\lim _{\Delta m_{21}^{2} \rightarrow 0} S_{\alpha \beta}^{\text {sol }}=0$.

The second condition, the completeness condition, demands that decomposition of the oscillation amplitude into the atmospheric and solar amplitudes be complete. We only have three neutrino states, and therefore, two independent $\Delta m^{2}$ 's: the atmospheric $\Delta m_{31}^{2}$ and the solar $\Delta m_{21}^{2}$. So, there should be two independent amplitudes, not more, and not fewer.

$$
\text { III. } \nu_{\mu} \rightarrow \nu_{e} \text { AND } \nu_{e} \rightarrow \nu_{e} \text { CHANNELS }
$$

To obtain a sense of what the atmospheric and solar amplitudes are, we write down their explicit forms in the $\nu_{\mu} \rightarrow \nu_{e}$ and $\nu_{e} \rightarrow \nu_{e}$ channels by using the flavor mixing matrix using the Particle Data Group (PDG) convention [5]. We leave the discussions of the other channels to Ref. [22].

The atmospheric and solar amplitudes, as defined in Eqs. (4) and (5), respectively, can be written in the $\nu_{\mu} \rightarrow \nu_{e}$ channel as

$$
\begin{aligned}
& S_{e \mu}^{\mathrm{atm}}=2 i s_{23} c_{13} s_{13} e^{-i \delta} e^{-i \frac{\Delta_{31} x}{2}} \sin \frac{\Delta_{31} x}{2}, \\
& S_{e \mu}^{\mathrm{sol}}=2 i s_{12} c_{13} e^{-i \frac{\Delta_{21} x}{2}}\left(c_{12} c_{23}-s_{12} s_{23} s_{13} e^{-i \delta}\right) \sin \frac{\Delta_{21} x}{2} .
\end{aligned}
$$

The oscillation probability consists of two terms, each amplitude squared and summed, and the interference term:

$$
P\left(\nu_{\mu} \rightarrow \nu_{e}\right)=\left|S_{e \mu}^{\mathrm{atm}}+S_{e \mu}^{\mathrm{sol}}\right|^{2} \equiv P_{\mu e}^{\mathrm{non}-\text { int-fer }}+P_{\mu e}^{\text {int-fer }},
$$

where

$$
\begin{aligned}
P_{\mu e}^{\text {non-int-fer }} \equiv & \left|S_{e \mu}^{\mathrm{atm}}\right|^{2}+\left|S_{e \mu}^{\mathrm{sol}}\right|^{2} \\
= & s_{23}^{2} \sin ^{2} 2 \theta_{13} \sin ^{2} \frac{\Delta_{31} x}{2}+\sin ^{2} \frac{\Delta_{21} x}{2} \\
& \times\left[c_{23}^{2} c_{13}^{2} \sin ^{2} 2 \theta_{12}+s_{23}^{2} s_{12}^{4} \sin ^{2} 2 \theta_{13}-8 s_{12}^{2} J_{r} \cos \delta\right], \\
P_{\mu e}^{\mathrm{int}-\mathrm{fer}} \equiv & 2 \operatorname{Re}\left[\left(S_{e \mu}^{\mathrm{atm}}\right)^{*} S_{e \mu}^{\mathrm{sol}}\right] \\
= & 8\left[J_{r} \cos \left(\delta+\frac{\Delta_{32} x}{2}\right)-s_{23}^{2} c_{13}^{2} s_{13}^{2} s_{12}^{2} \cos \left(\frac{\Delta_{32} x}{2}\right)\right] \\
& \times \sin \frac{\Delta_{21} x}{2} \sin \frac{\Delta_{31} x}{2} .
\end{aligned}
$$


We note that the interference term, the second equation of Eq. (10), displays the key feature of the problem. That is, it consists of two terms: one that depends on $\delta$ and another that does not. The ability to observe the effect of $\delta$ comes from the quantum interference between the atmospheric and solar amplitudes, but it is only part of the interference effect. A claim of observation of the quantum interference between the atmospheric and the solar amplitudes requires the observation of both terms in Eq. (10) with the correct magnitudes; i.e., a measurement of $\delta$ is not the same as a measurement of the interference effect.

Now, we discuss the $\nu_{e} \rightarrow \nu_{e}$ channel, which is identical to the $\bar{\nu}_{e} \rightarrow \bar{\nu}_{e}$ channel due to $C P T$ invariance. The atmospheric and solar amplitudes are written as

$$
\begin{aligned}
& S_{e e}^{\mathrm{atm}}=2 s_{13}^{2} e^{-i \frac{\pi}{2}} e^{-i \frac{\Delta_{31} x}{2}} \sin \frac{\Delta_{31} x}{2}, \\
& S_{e e}^{\mathrm{sol}}=2 s_{12}^{2} c_{13}^{2} e^{-i \frac{\pi}{2}} e^{-i \frac{\Delta_{21} x}{2}} \sin \frac{\Delta_{21} x}{2} .
\end{aligned}
$$

Due to the unoscillated 1 in Eq. (6), the $\nu_{e}$ survival probability $P\left(\nu_{e} \rightarrow \nu_{e}\right)$ takes a slightly complicated form, but it can be written in a similar form as in the appearance channel:

$$
P\left(\nu_{e} \rightarrow \nu_{e}\right)=P_{e e}^{\text {non-int-fer }}+P_{e e}^{\text {int-fer }},
$$

where

$$
\begin{aligned}
P_{e e}^{\text {non-int-fer }} \equiv & 1+\left|S_{e e}^{\mathrm{atm}}\right|^{2}+\left|S_{e e}^{\mathrm{sol}}\right|^{2}+2 \operatorname{Re}\left[S_{e e}^{\mathrm{atm}}+S_{e e}^{\mathrm{sol}}\right] \\
= & 1-\sin ^{2} 2 \theta_{13} \sin ^{2} \frac{\Delta_{31} x}{2} \\
& -4 s_{12}^{2} c_{13}^{2}\left(1-s_{12}^{2} c_{13}^{2}\right) \sin ^{2} \frac{\Delta_{21} x}{2}, \\
P_{e e}^{\text {int-fer }} \equiv & 2 \operatorname{Re}\left[\left(S_{e e}^{\mathrm{atm}}\right)^{*} S_{e e}^{\mathrm{sol}}\right] \\
= & 2 \sin ^{2} 2 \theta_{13} s_{12}^{2} \sin \frac{\Delta_{31} x}{2} \cos \frac{\Delta_{32} x}{2} \sin \frac{\Delta_{21} x}{2} .
\end{aligned}
$$

\section{HOW TO OBSERVE THE QUANTUM INTERFERENCE EFFECT}

We briefly discuss how to pin down the quantum interference effect between the atmospheric and solar amplitudes. Once we obtain the expression of the oscillation probability as

$$
P\left(\nu_{\beta} \rightarrow \nu_{\alpha}\right)=P_{\beta \alpha}^{\text {non-int-fer }}+P_{\beta \alpha}^{\text {int-fer }},
$$

we can define a "test oscillation probability" by introducing the $q$ parameter as

$$
P\left(\nu_{\beta} \rightarrow \nu_{\alpha}\right)=P_{\beta \alpha}^{\text {non-int-fer }}+q P_{\beta \alpha}^{\text {int-fer }} .
$$

By fitting the data with the test oscillation probability [Eq. (15)], we would obtain a 1-dimensional $\chi^{2}$ (1 DOF) for the $q$ parameter. We note that, in the case of appearance experiments, we marginalize over $\delta$ as well as the other mixing parameters in the experimentally allowed ranges.

Though our discussion in this paper covers both the appearance and the disappearance experiments in vacuum, the analysis of the appearance channel in accelerator LBL experiments requires treatment of the matter effect [22], which is beyond the scope of this paper.

The experimental setting of JUNO [23] is uniquely suited for our purpose of observing the interference effect between the atmospheric and solar oscillations. In JUNO, the solar and atmospheric oscillation effects coexist with their full magnitudes at the same detector. Both oscillations are fully developed and have left the linear regime of $\sin \frac{\Delta_{k 1} x}{2}$. Even though the atmospheric oscillation may be small wiggles over the long-wavelength solar oscillation, the very good energy resolution of the JUNO detector aims at its precision measurement. Therefore, JUNO is an ideal experiment for the purpose of detecting the atmospheric-solar interference effect. It is very likely the best choice among all possible experiments, ongoing or planned, in vacuum and in matter.

Here, we describe in detail the procedure of our statistical analysis. Using GLoBES [24,25], we set up an experiment with two detectors: a JUNO-like far detector with a fiducial mass of $20 \mathrm{kt}$ and an energy resolution of $3 \% / \sqrt{E}$ at a distance of $53 \mathrm{~km}$ from a nuclear reactor source with a total thermal power of $36 \mathrm{GW}_{\text {th }}$, and a TAOlike [26] near detector with a fiducial mass of 1 ton and an energy resolution of $1.7 \% / \sqrt{E}$ at a distance of $30 \mathrm{~m}$ from a $4.6 \mathrm{GW}_{\text {th }}$ nuclear reactor core; we assume a total datataking time of 6 years. For each detector, we use a model for nonlinear effects in the reconstruction of the positron energy like that described in Ref. [27] up to cubic terms. To account for the uncertainties in the reactor antineutrino flux prediction, we conservatively introduce a nuisance parameter to each of our 100 energy bins with the spectrum computed before applying the energy resolution function. This is equivalent to the assumption of no prior knowledge of fluxes, as in Ref. [27]. For the purposes of producing simulated data, we assume the normal ordering to be the true mass ordering and the relevant oscillation parameters to be $\Delta m_{21}^{2}=7.54 \times 10^{-5} \mathrm{eV}^{2}, \Delta m_{31}^{2}=2.43 \times 10^{-3} \mathrm{eV}^{2}$, $\theta_{12}=33.6^{\circ}$, and $\theta_{13}=8.9^{\circ}$. For the analysis of the resulting data, we fit the data obtained from the oscillation probability in Eq. (13) with those obtained using the oscillation probability modified with the parameter $q$, as in Eq. (15), by minimizing the following $\chi^{2}$ function for various values of $q$ while allowing all nuisance and standard oscillation parameters to vary:

$$
\chi^{2}=\sum_{i, I} \frac{\left(\phi_{\mathrm{true}, i}^{I}-\phi_{\mathrm{fit}, i}^{I}\right)^{2}}{\phi_{\mathrm{true}, i}^{I}}+\text { pull terms }
$$

where $\phi_{\text {true }, i}^{I}$ and $\phi_{\mathrm{fit}, i}^{I}$ are the simulated rate and modified rate, respectively, in the $i$ th energy bin for the detector 
specified by $I=$ Near, Far. The "pull terms," defined in Eq. (17), provide a penalty for $\theta_{13}$ with an uncertainty of $\sigma_{\theta_{13}}=10 \%$ and the nuisance parameters $n_{k}$ for which uncertainties are $\sigma_{k}$ :

$$
\text { pull terms }=\frac{\left(\theta_{13, \text { true }}-\theta_{13, \text { fit }}\right)^{2}}{\sigma_{\theta_{13}}^{2}}+\sum_{k} \frac{n_{k}^{2}}{\sigma_{k}^{2}}
$$

The nuisance parameters included in the "pull terms" encode the uncertainties for energy calibration (only linear terms), fiducial mass of each detector, and flux, as described in detail in Ref. [27].

The resulting $\chi^{2}$ curve is shown as the thick black line in Fig. 1 . At $q=0$, the value of $\chi^{2}$ is 16.7 , so the interference effect would be able to be seen in JUNO with a significance of more than $4 \sigma$.

The same analysis procedure is repeated, with the assumption that the energy calibration error for each detector is linear (blue solid line), and then without a near detector while assuming perfect knowledge of the detector and source systematics (gray dashed line).

Note that there is a potential model dependence, in that we assume that atmospheric oscillation experiments observe $\Delta m_{31}^{2}$. If, instead, we assume that they measure $\Delta m_{32}^{2}$, the value of $\chi^{2}$ at $q=0$ for the scenario with a near detector and the most conservative systematics is still 16.7.

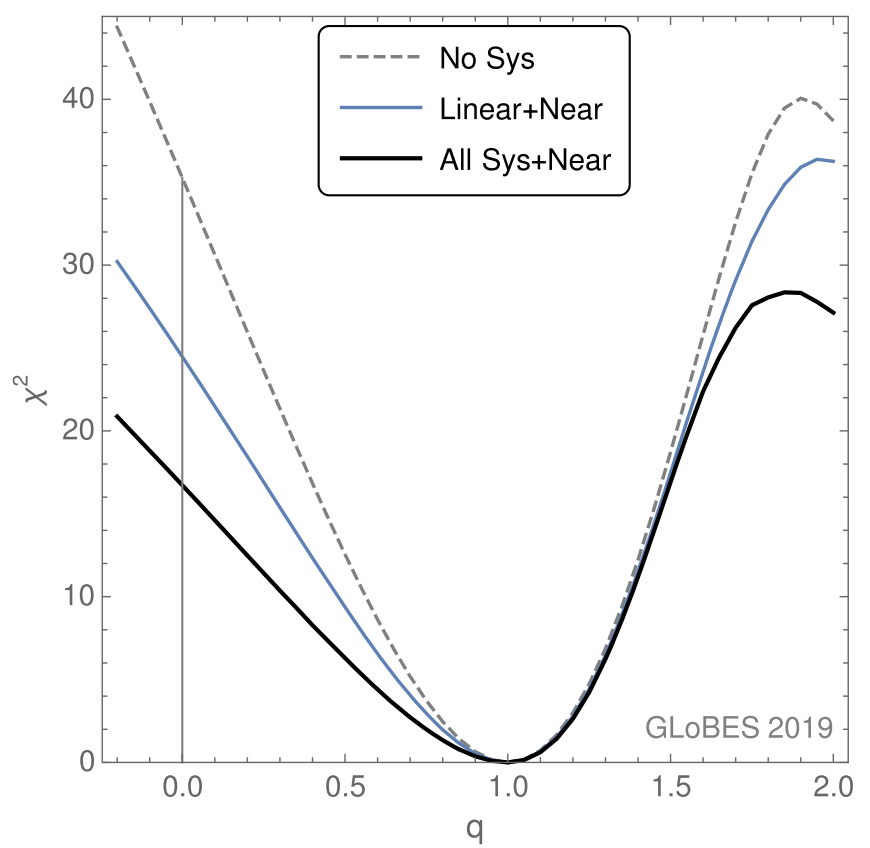

FIG. 1. $\chi^{2}$ as a function of the $q$ parameter introduced in Eq. (15). The thick solid line includes all systematics with a near detector, whereas the blue line assumes the energy-scale errors are linear. The gray dashed line is computed without any systematics and with the assumption of a perfect knowledge of the initial neutrino flux and no near detector.

\section{INTERFERENCE EFFECT AND MASS ORDERING}

It is a natural question to ask how the sensitivity to the interference effect depends on the neutrino mass ordering, and conversely, whether the capability to determine the mass ordering is due to the interference between the atmospheric and solar waves. Hereafter, we use the abbreviations "NO" and "IO" for the normal and the inverted orderings, respectively.

We start by recalling that the interference term, the second term in Eq. (13), must be the origin of sensitivity to the mass ordering, because it is the only term which is odd under the inversion NO $\leftrightarrow \mathrm{IO}$. This seems to support the notion expressed in Refs. $[19,20]$ that the mass-ordering resolution is due to the interference between the atmospheric-solar waves. However, we will show that the reality is a little more complicated. ${ }^{3}$

The mass-ordering dependence of $\chi^{2}(q)$ is examined in Fig. 2 using the most conservative systematics and including a near detector, as in the previous section. If we use the NO (IO) for both the true mass ordering and the test probability for fitting, the black solid (blue dotted) curve results. These two curves indicate that sensitivity to the interference does not depend on the mass ordering.

The behavior of $\chi^{2}(q)$ drastically changes if the JUNO data are fit with the wrong mass ordering. In Fig. 2, the blue solid (black dotted) curve is the case of the true $\mathrm{NO}(\mathrm{IO})$ fit with the IO (NO) hypothesis. The large value of $\chi^{2}(q) \approx 25$ at $q=1$ tells us that JUNO can refute the wrong mass ordering at a confidence level around $5 \sigma$ in both cases of the true mass orderings. This result is consistent with the one in Ref. [27]. The precise value depends on the details of the systematics implementation-see also Ref. [23] and the references cited therein - but this is not germane.

One might naively expect that detection of the interference term [Eq. (13)] would be trivial for JUNO, as it is large, $\sim \sin ^{2} 2 \theta_{13} \simeq 0.1$. However, this sensitivity is being reduced by a combination of not knowing the values of the oscillation parameters precisely enough to start with and cancellation occurring due to the energy bins. In fact, one can show analytically that an integration of the atmospheric-scale oscillation of $P_{e e}^{\text {int-fer }}$ in Eq. (13) over $\frac{1}{4}$ of a period cancels the contribution from the adjacent $\frac{1}{4}$ period under the approximation $\sin \Delta_{21} x \sim \Delta_{21} x$. Then, it can be translated into the cancellation among different energy bins in integration over $1 / E$, which leads to an imperfect but efficient cancellation in energy space. It is conceivable that such cancellation contributes substantially to the behavior of $\chi^{2}(q)$ in the right and wrong mass-ordering fits.

Another key feature of the question is the minimum of $\chi^{2}(q)$ at $q \simeq 2$ in the wrong mass-ordering fit. It is produced

\footnotetext{
${ }^{3}$ Note also that the interference term defined in Refs. $[19,20]$ is different from ours.
} 


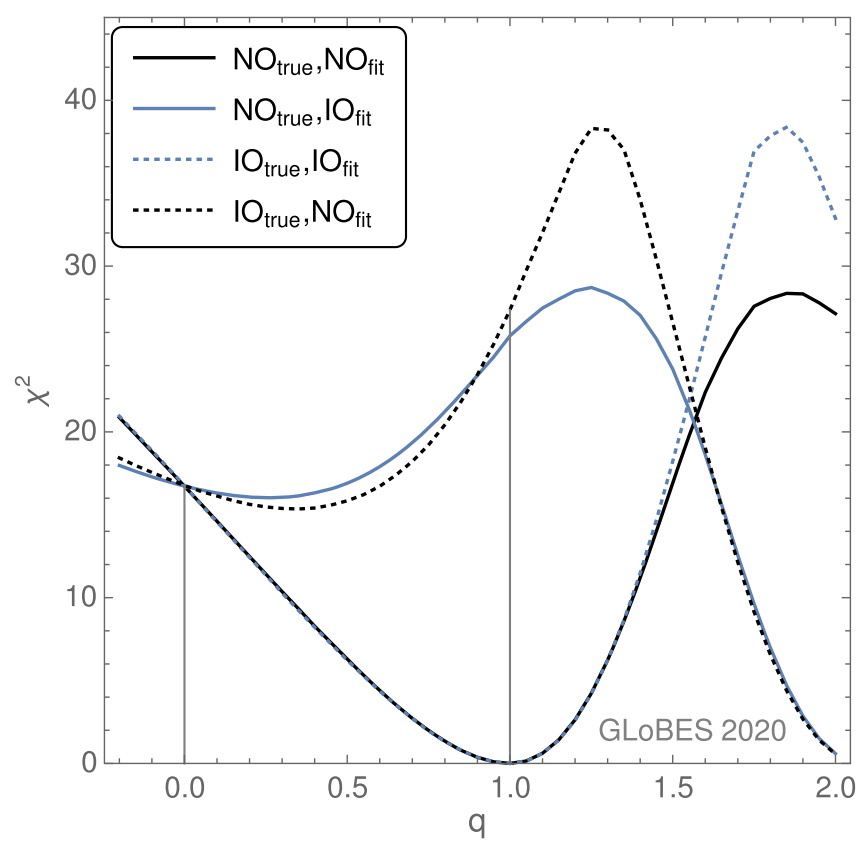

FIG. 2. $\chi^{2}$ as a function of the $q$ parameter defined in Eq. (15) with all the systematics and with a near detector. The solid lines are for the case in which the true mass ordering is normal, which are fit with the hypotheses of the NO (black line) or IO (blue line). The dotted lines are for the case of true IO, which are fit with the NO (black line) or IO (blue line) hypotheses. The black solid curve is identical with the one in Fig. 1.

by allowing both $\theta_{12}$ and $\Delta m_{31}^{2}$ to float freely in the fit. If we instead kept all the oscillation parameters fixed, we would find that all of the curves become parabolic with their minimum at $q \simeq 1$, albeit with the wrong massordering fit curves having a much larger value at the minimum. The minimum around $q=2$ arises as a combined effect of shifting $\Delta m_{31}^{2}$ by about $1 \%$ from its input value and $\sin ^{2} \theta_{12}$ by about $3 \%$, respectively. The occurrence of this minimum is independent of systematics and energy resolution, which can be demonstrated by using a pseudo- $\chi^{2}, \hat{\chi}^{2}$, defined as

$$
\hat{\chi}^{2}(q)=\int d E\left[P_{\mathrm{NO}}(E, q=1)-P_{\mathrm{IO}}(E, q)\right]^{2} .
$$

Apart from an overall scale factor, $\hat{\chi}^{2}$ reproduces the $q$ dependence shown in Fig. 2 if $\theta_{12}$ and $\Delta m_{31}^{2}$ are allowed to float. Moreover, we find that the value of $q$ for which the IO minimum occurs scales like $\sin ^{-2} \theta_{12}$. We conclude that this second minimum is due to a complete cancellation at the probability level by accident-i.e., the values of oscillation parameters it occurs at do not represent an intrinsic symmetry of the oscillation probability. This rather striking behavior also demonstrates that a determination of the mass ordering, which entirely takes place at $q=1$, is not equivalent to studying the question of whether the $q$ term (i.e., the interference term) is present at all.
The origin of the local deep minimum of $\chi^{2}(q)$ at $q \sim 2$ can also be understood by following the analysis technique outlined in Ref. [21], which is using the Fourier transform of the event spectrum or probability as a function of $L / E{ }^{4}$ In the Fourier spectrum, one observes a main peak at $\Delta m_{31}^{2}$ with a shoulder at $\Delta m_{31}^{2} \pm \Delta m_{21}^{2}$, and the mass hierarchy is determined by the relative positions of the shoulder and the main peak. Note that the absolute positions of the peaks are only known within the uncertainty of $\Delta m_{31}^{2}$, which is much larger than their separation.

Varying $q$ changes the relative amplitudes of the peaks and can thus lead to a confusion of mass ordering. If we use $\hat{\chi}^{2}$ in Eq. (18) with the true NO, which has a local minimum at $q \simeq 2$, the relative positions of the higher and lower peaks exchange when $q$ is increased from $q=1$ and $q \simeq 2$.

Conceptually, it is possible to imagine a world in which $\Delta m_{31}^{2}$ is determined with superb accuracy $\left(\ll \Delta m_{21}^{2}\right)$. In this case, one can show numerically that the degeneracy indeed goes away, and hence there is no confusion, since now the positions of the peaks, instead of their relative heights, can be used to determine the mass hierarchy. Thus, in that case, the sensitivity to mass ordering exists in a robust way independent of the potential strength of the interference term.

To summarize, we failed to see evidence for an intimate, direct connection between sensitivity to the mass ordering and the atmospheric-solar interference effect.

\section{SUMMARY}

In this paper, we have shown that, in vacuum, a natural and general way of decomposing the oscillation amplitudes into solar and atmospheric parts is possible for the appearance and disappearance channels. This decomposition is exact and relies neither on the hierarchical values of the two $\Delta m^{2}$ nor on the actual values of observed oscillation parameters. With this amplitude decomposition, it becomes possible to define the effect of interference between the two partial amplitudes. For appearance channels, the interference term contains the $C P$ phase $\delta$, but also terms independent of it.

In the $\bar{\nu}_{e}\left(\nu_{e}\right)$ disappearance channel, the oscillation amplitude does not depend on $\delta$, and hence, the interference effect we saw has nothing to do with the $C P$ phase. The nature of the interference phenomena indicated by these features is a dynamical, quantum mechanical interference inside the three families of neutrinos, not particularly related to the $C P$-violating phase. We show, by detailed numerical calculation, that JUNO can observe this interference effect with more than $4 \sigma$ significance.

\footnotetext{
${ }^{4}$ Note that the Fourier approach, while conceptually very clear, is not well suited to a full study including systematic effects on the energy scale, and thus it is not used for actual sensitivity estimates; see, for example, Ref. [23].
} 
We have also discussed the relationship between the interference effect and sensitivity to the mass-ordering resolution. We argued that though the latter comes from the interference term in Eq. (13), we see no supporting evidence for the hypothesis of the mass-ordering resolution being equivalent to the atmospheric-solar wave interference effect.

\section{ACKNOWLEDGMENTS}

One of the authors (H. M.) thanks Takaaki Kajita and Hiroshi Nunokawa for intriguing conversations while this project was still in its infancy. The work of P. H. and R. P. is supported by the U.S. Department of Energy Office of Science under Award No. DE-SC0020262.

Note added.-We have recently learned that the author of Ref. [28] used unitarity as in Eq. (4) to derive the alternative form of the oscillation probability in the $N$-flavor case which agrees with our formula for $N=3$. However, neither the physics of atmospheric-solar interference, nor the amplitude decomposition with completeness is discussed in that paper.
[1] J. H. Christenson, J. W. Cronin, V. L. Fitch, and R. Turlay, Phys. Rev. Lett. 13, 138 (1964).

[2] M. Kobayashi and T. Maskawa, Prog. Theor. Phys. 49, 652 (1973).

[3] B. Aubert et al. (BABAR Collaboration), Phys. Rev. Lett. 87, 091801 (2001).

[4] K. Abe et al. (Belle Collaboration), Phys. Rev. Lett. 87, 091802 (2001).

[5] M. Tanabashi et al. (Particle Data Group), Phys. Rev. D 98, 030001 (2018).

[6] E. Majorana, Nuovo Cimento 14, 171 (1937).

[7] K. Abe et al. (T2K Collaboration), Nature (London) 580, 339 (2020).

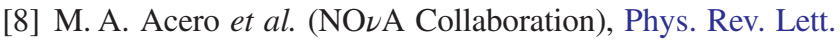
123, 151803 (2019).

[9] M. Jiang et al. (Super-Kamiokande Collaboration), Prog. Theor. Exp. Phys. 2019, 053F01 (2019).

[10] K. Abe et al. (Hyper-Kamiokande Proto-Collaboration), Prog. Theor. Exp. Phys. (2015), 053C02.

[11] R. Acciarri et al. (DUNE Collaboration), arXiv:1512.06148.

[12] Y. Fukuda et al. (Super-Kamiokande Collaboration), Phys. Rev. Lett. 81, 1562 (1998).

[13] K. Eguchi et al. (KamLAND Collaboration), Phys. Rev. Lett. 90, 021802 (2003).

[14] L. Wolfenstein, Phys. Rev. D 17, 2369 (1978).
[15] S. P. Mikheyev and A. Y. Smirnov, Yad. Fiz. 42, 1441 (1985) [Sov. J. Nucl. Phys. 42, 913 (1985)].

[16] Q. R. Ahmad et al. (SNO Collaboration), Phys. Rev. Lett. 89, 011301 (2002).

[17] A. Y. Smirnov, arXiv:hep-ph/0610198.

[18] E. K. Akhmedov, M. Maltoni, and A. Y. Smirnov, J. High Energy Phys. 06 (2008) 072.

[19] S. T. Petcov and M. Piai, Phys. Lett. B 533, 94 (2002).

[20] S. Choubey, S. T. Petcov, and M. Piai, Phys. Rev. D 68, 113006 (2003).

[21] J. Learned, S. T. Dye, S. Pakvasa, and R. C. Svoboda, Phys. Rev. D 78, 071302 (2008).

[22] P. Huber, H. Minakata, and R. Pestes (to be published).

[23] F. An et al. (JUNO Collaboration), J. Phys. G 43, 030401 (2016).

[24] P. Huber, M. Lindner, and W. Winter, Comput. Phys. Commun. 167, 195 (2005).

[25] P. Huber, J. Kopp, M. Lindner, M. Rolinec, and W. Winter, Comput. Phys. Commun. 177, 432 (2007).

[26] J. P. A. M. de André for JUNO, Physics prospects of JUNO, Talk at The 27th International Workshop on Weak interactions and Neutrinos (WIN2019), Bari, Italy, June 3-8 (2019)

[27] D. V. Forero, R. Hawkins, and P. Huber, arXiv:1710.07378.

[28] S. M. Bilenky, arXiv:1208.2497. 